\title{
Phronesis
}

\section{Le stage en formation}

Tendances et résistances

\section{Philippe Maubant, Emmanuel Triby et Souâd Denoux}

Volume 3, numéro 1-2, janvier-avril 2014

Le stage en formation, tendances et résistances

URI : https://id.erudit.org/iderudit/1024583ar

DOI : https://doi.org/10.7202/1024583ar

Aller au sommaire du numéro

Éditeur(s)

Université de Sherbrooke

ISSN

1925-4873 (numérique)

Découvrir la revue

Citer ce document

Maubant, P., Triby, E. \& Denoux, S. (2014). Le stage en formation : tendances et résistances. Phronesis, 3(1-2), 1-5. https://doi.org/10.7202/1024583ar d'utilisation que vous pouvez consulter en ligne.

https://apropos.erudit.org/fr/usagers/politique-dutilisation/ 


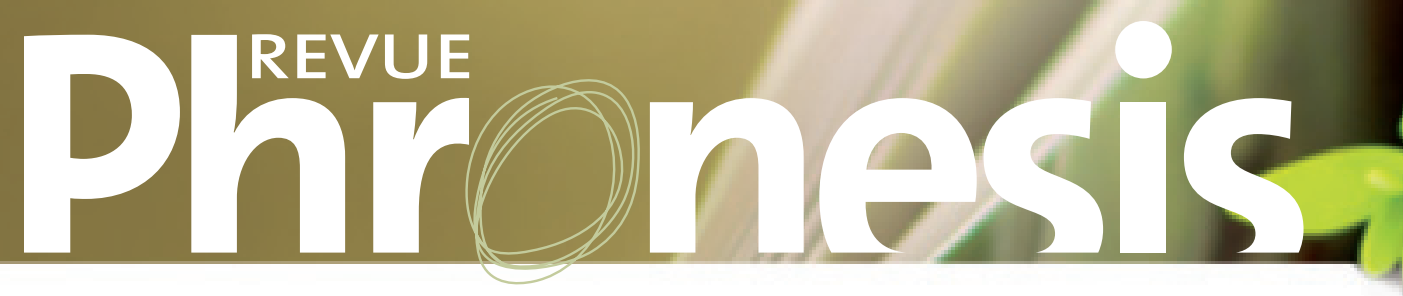

\section{Le stage en formation}

Philippe MAUBANT*, Emmanuel TRIBY**, Souâd DENOUX***

*Université de Sherbrooke, Canada CRIFPE-Sherbrooke

2500 boul de l'Université

Sherbrooke, Qc, Canada

J1K $2 R 1$
${ }^{* *}$ LISEC

Université de Strasbourg

4 Rue Blaise Pascal,

67400 Strasbourg, France
${ }^{* * *}$ LIRDEF,

Université Montpellier 3

Route de Mende,

34199 Montpellier, France

\section{Tendances et résistances}


Le cheminement de ce dossier sur la «stagification » permet d’en montrer à la fois l'intérêt et les enjeux. Rappelons que la référence à l’article historique de Jacques Guigou - La stagification, Éducation permanente, 1975 - signifiait notre souci de travailler sur l'objet même de cet article : le devenir de la formation des adultes, particulièrement sous l'espèce de la formation professionnelle continue (FPC).... Et si nous pensions que le choix de cet objet s'imposait, cétait parce qu'il n'avait pas vraiment fait l'objet d'investigations savantes, ou alors de façon éparse, dispersée, peut-être même anecdotique. Et si la «forme » stage appliquée à la formation des adultes avait été si peu ou si mal travaillée, cétait sans doute parce quelle ne méritait pas l'attention des chercheurs... L'objet du dossier s'imposait donc, mais comment le nourrir ? En effet, s'il y a une littérature abondante dans les revues professionnelles du secteur, les chercheurs semblent moins intéressés par les modalités de développement de la formation des adultes : organisation du temps et de lespace de la formation, structuration des démarches en situation, nature des savoirs effectivement valorisés...

La proposition de collaboration faite par la revue canadienne Phronésis a changé radicalement lorientation du dossier et du même coup a limité le risque d'un défaut de contributions de qualité : la stagification oui, mais partout ! Tous les stages, y compris en formation initiale, dans des dispositifs de formation par alternance et dans les formations générales... Ce faisant, pourtant, acceptant cette ouverture, notre préoccupation pour la forme «stage » en FPC perdait de son importance; elle mériterait pourtant des investigations plus poussées.

En formation des adultes, en effet, le stage représente une organisation dominante de loffre de formation, notamment en formation professionnelle continue, mais également pour les demandeurs d'emploi. Il correspond à une "petite forme ", telle qu'on la nomme dans le domaine du spectacle vivant : durée limitée, public restreint, infrastructure très légère, effectif faible d'intervenants plutôt polyvalents et grande reproductibilité dans des contextes variés. Ces stages cohabitent aujourd'hui avec d’autres modes dorganisation du temps de la formation : formations diplômantes « longues», formation en situation de travail...

La stagification est le terme promu par Jacques Guigou dans les années 1970 pour nommer l'apparition et l'amplification de cette modalité particulière dorganisation de la formation (Guigou, 1975). Depuis lors, les stages courts ont confirmé leur place dans la formation professionnelle continue. Parmi les tendances actuelles en formation d'adultes, le stage occupe clairement la fonction d'indicateur d'activité de la branche, «l'unité de compte absolue des activités de formation permanente ».

Le poids de léconomique semble important dans lémergence et la dissémination des stages. Cette prégnance doit être relevée, notamment en rapport aux exigences de la relation pédagogique. Aujourd'hui, le jeu de léconomique apparaît sous deux aspects au moins : une logique de produit sur un marché qui, du fait même de l'extrême diversité des offreurs et de léparpillement des capacités de financement, a besoin de caractéristiques relativement stables ou stabilisées pour attirer une demande ; une logique de processus, ou de production, quand il s'agit de réaliser une sorte de rationalisation de l'acte de transmission afin de répondre à certaines contraintes, tant matérielles et financières que pédagogiques. Le développement de l'ingénierie de formation fonctionne ici à la fois comme effet et source d'expansion de cette double dynamique.

Cette « petite forme » du stage semble autoriser une réponse plus fine aux besoins de formation des personnes ; souvent décrit comme un nouveau mode pédagogique porteur d'une dimension intrinsèquement formative, le stage permettrait un certain équilibre entre l'appris, l'expérimenté et le professionnel. À ce titre, il trouve notamment toute sa place dans la conception et l'organisation des formations par alternance dont il constitue la composante traditionnellement emblématique. Il est le «terrain » qui empêcherait les formations professionnelles de sombrer dans la «théorie ", ou à des formations plus " générales " de ne pas oublier quelles sont faites pour s’investir un jour dans de « vraies » situations professionnelles...

Les organisations marchandes, comme les collectivités responsables de la formation des demandeurs d'emploi, y voient également la possibilité d'une gestion plus rationnelle et responsable des besoins de formations (Boutinet, 2011) : le stage trouverait naturellement sa place dans des temporalités très contraintes et une forte différenciation des demandes des partenaires concernés par la formation. Enfin, une 
offre de formation plus standardisée serait forcément moins coûteuse : entre appels d'offre et cahiers des charges, en rapport aux exigences des démarches qualité et de la certification, le stage s’inscrirait facilement dans la «chaîne de valeur » des organisations marchandes ou dans les dispositifs de gestion du chômage et leur justification politique.

Sur le terrain, la multiplication des stages courts a largement concouru à la promotion de lévaluation "à chaud ", alors que leur impact effectif reste flou et finalement encore peu recherché par maints financeurs ; cela a peut-être participé au vigoureux mouvement de formalisation qui caractérise aujourd'hui la formation : scénarisations, dispositifs collaboratifs, référentiels, outils de suivi et dévaluation... Un autre effet majeur de cette ingénierie de plus en diversifiée dans ses objets et ses démarches est la construction d'un marché de " produits " de la formation : la mise en forme stage est apparemment une condition pour accéder à de nouvelles modalités de rationalisation de l'activité de formation. La stagification a également favorisé la prédominance des formations d’adaptation au détriment des formations qualifiantes. Enfin, ce phénomène a accéléré l'inscription des temps de la formation dans le temps de travail du salarié (Lambert et alii, 2009), avec le risque de diminuer le temps effectivement accordé à la formation.

Le devenir probable des stages se joue sans doute dans la différenciation de leurs modalités de fonctionnement. Les nouvelles lois régissant la FPC - le DIF notamment et sa "portabilité » - pourraient accélérer le mouvement de stagification tel que nous l'entendons. De même, le rôle croissant que joue aujourd'hui la certification dans la formation et dans le processus de professionnalisation risque de pousser à laccroissement du nombre de stages courts. Des questions sont ouvertes : quelles seront les incidences du recours aux TIC et celles des nouvelles modalités d’organisation de la formation (portabilité du DIF, mutualisation, modularisation..) ? Lindividualisation en cours renforcera-t-elle la tendance à la stagification ? La forte dynamique de la FOAD et la préférence pour la mise en situation professionnelle vont-elles signer la mort du stage ou simplement sa transformation?

En somme, plus de 40 ans après la montée en puissance de cette " petite forme », suite à la loi de 1971 notamment, les stages restent au cœur de la formation des adultes. Celle-ci se transforme profondément en favorisant à la fois leur multiplication et leur diversification. Compte tenu de cette apparente généralisation selon des modalités de plus en plus différenciées, il est aujourd'hui nécessaire de faire le point sur la signification actuelle et les enjeux à venir de cette organisation de la formation.

En tant que composante d'un parcours de formation initiale, le stage suscite actuellement un grand intérêt en France chez les responsables politiques, dans les entreprises, dans les formations universitaires et les écoles d’enseignement supérieur, chez les professionnels de l'éducation et de la formation ainsi que chez les sujets en formation (apprenants, étudiants, élèves,...). Sa grande visibilité sociale conforte sa place dans le paysage éducatif et conduit les milieux scientifiques et de la recherche à s’y intéresser de façon plus intense. En témoignent des journées détudes, en France ou au Québec, des colloques organisés sur ce thème notamment à l'École Nationale des Ponts, à l'Université Paris-Est Créteil, l'existence de réseaux, à l'instar du RESTAG (Réseau d'Étude sur les Stages et leur Gouvernance), les différentes publications et des propositions de projets ANR en cours, mobilisant de nombreux chercheurs de différentes institutions (CNAM, LARGOTEC, LISEC, $\mathrm{ETE}, \ldots)$.

Dans le contexte actuel, les stages constituent une réponse possible à l'injonction sociale et économique d'employabilité, de développement des compétences et de professionnalisation. Ils représentent souvent la première expérience professionnelle, au sens le plus large : travail en vraie grandeur pouvant faire découvrir différents aspects du monde professionnel. Ils se caractérisent par la diversité de leurs objectifs : stage de découverte, stage ouvrier, stage de probation,... ainsi que par celle du statut octroyé au stagiaire : stagiaire de la formation professionnelle, étudiant, élève, apprenti. Ils marquent peut-être surtout le souci des organisations, financièrement et budgétairement de plus en plus contraintes, de se défausser du coût du temps d’apprentissage.

La forte présence des stages dans l'actualité souligne qu'ils constituent une question sociale vive, pendant les études et, de plus en plus, dans les phases dites de professionnalisation et/ou de « transition ». Le phénomène est particulièrement net dans les universités au sein desquelles le stage constitue loutil essentiel de ce qu'il est convenu d’appeler leur professionnalisation (Combes, 2011), occasion de (ou prétexte pour) repenser leur fonction d'insertion et leur partenariat avec les entreprises. Il concerne aussi les « formations insertion » destinées aux jeunes dits « en difficulté », où il a une fonction d'accès au marché du travail. Faisant l'objet d'un accompagnement très rapproché, il s'apparente à une mise au travail fort différente de l'extraction et de la suspension hors du travail que recouvre le stage d'adultes en FPC.

Notre projet a été de proposer un dossier sur les stages simultanément dans deux revues Éducation et socialisation et Phronésis. Ces deux revues sont intéressées par les questions de professionnalisation et il nous a semblé important de présenter conjointement des recherches en provenance des "deux côtés de l'Atlantique ", mais surtout de mettre à disposition et de rapprocher un ensemble de contributions aux orientations et aux objets divers mais finalement très convergents ; une analyse attentive fera apparaitre que la formulation des questions, les préoccupations pratiques, les notions clés pour construire les problématiques et l'intelligibilité, sont quand même différentes. 
Notre dossier ne peut avoir la prétention d’aborder de front les questions soulevées par la multiplication des stages en formation initiale, notamment à travers la place occupée par la dite " alternance » dans la quasi totalité des filières scolaires et universitaires (Merle et Théry, 2012 ; Briant et Glaymann, 2013). En revanche, pour enrichir notre problématique, il est important de s'intéresser particulièrement à deux objets de questionnement :

- en premier lieu, en quoi les visées propres des stages en formation initiale se distinguent-elles de celles de la formation continue ? Nous portons notamment notre attention sur leur place, supposée et réelle, dans la constitution des capacités professionnelles, ainsi que le sens et la portée effective de la situation d’apprentissage dans ces périodes particulières.

- en second lieu, quels liens est-il ou serait-il possible détablir entre la formation initiale et la formation continue en la matière, pour en développer l'efficacité pédagogique et professionnelle ? Nous relèverons notamment la place des stages dans les parcours d'insertion et de mobilité des personnes dans des secteurs particuliers (enseignement, santé, travail social,...), l'exercice de lévaluation des acquis et sa continuité éventuelle entre les deux «niveaux » de formation.

En ouverture du dossier, Triby propose de revenir sur l'article « historique » qui en a été en quelque sorte le déclencheur. Clairement situé dans une conjoncture idéologique et politique particulière, larticle n’en comporte pas moins une perspective théorique sur la formation des adultes, ou plus précisément sur la théorie des «formes » en éducation; avant la « forme scolaire » identifiée par Guy Vincent et son équipe quelque 20 ans plus tard, Jacques Guigou avait identifié la « forme » prise par la formation des adultes sous la modalité archétypique du stage court, standardisé, centré sur un objet consommable, en somme industrialisé.

Presquà l'opposé de cette organisation fordiste de la formation, Denoux propose détudier les conditions de l'apprentissage en stage par une sorte de modélisation de lactivité apprenante en situation, modèle quelle confronte aux modalités concrètes de fonctionnement et d’acquisition de compétences lors de stages de formation de conducteurs de travaux dans le secteur du BTP.

Poursuivant l'analyse des conditions d’une efficacité pédagogique des stages, Roger, Jorro et Maubant relèvent l'importance de centrer la formation sur les situations dont le stage ne constitue qu'une modalité possible ; c'est la diversité des situations qui détermine la portée d'un processus de formation, comme autant d’occasions de produire une expérience. Dans cette perspective, un certain nombre de dispositions doivent accompagner ces "mises en situation ", notamment lécriture, passage obligé pour accéder à lémergence d'une réflexivité au fondement même de l'apprentissage durable et la professionnalité.

Deux articles présentent ensuite des études sur les stages dans la formation des formateurs. Revenant sur l'importance des traces pour construire des apprentissages, Eneau, Lameul et Bertrand s'intéressent tout particulièrement à l'usage du port folio dans l'accompagnement d'une formation, notamment à distance, du fait des interactions que cet outil autorise entre les différentes parties prenantes d'une formation impliquant des stages. Savarieau revient sur l'importance de laccompagnement, particulièrement en FOAD, mais en se centrant sur la mission dévolue au stagiaire, notamment quand le stagiaire, formateur en formation, fait son stage dans sa propre entreprise : l'analyse de contenu de lettres de mission entre en résonnance avec les modalités d’un accompagnement à distance de la mise en œuvre de ces missions. En somme, les stages ne peuvent trouver leur plein accomplissement en termes de formation que dans des modalités particulières de l'accompagnement des stagiaires.

Deux autres contributions développent la question très actuelle de la multiplication des stages dans les cursus universitaires. A partir des résultats d'une recherche-action de longue durée, Glaymann relève le faible impact des stages sur l'insertion des étudiants ; pour autant, ces stages gardent une forte utilité en matière de socialisation professionnelle des jeunes étudiants, notamment dans les filières des sciences humaines et sociales. L'article complète ainsi l'effort de modélisation en tentant d'identifier les caractéristiques du «bon stage ». Patroucheva centre davantage son analyse sur les points de vue croisés des universitaires et des employeurs pour faire apparaitre la distance entre ces deux partenaires de la mise en stage des étudiants, une distance paradoxalement liée à une conception très adéquationniste de la formation. En somme, c'est la dimension économique des stages en formation initiale qui est ainsi analysée, du point de vue des entreprises comme des stagiaires, en termes d'impacts (sur l’emploi et les coûts), mais également dans la mise en œuvre effective d'intentions formatives.

Trois articles closent le dossier en s'intéressant à la place des stages dans la formation des enseignants. Boutet et Villemin reviennent sur l'importance de laccompagnement qui fait si gravement défaut aux stages des cursus universitaires, en permettant la construction progressive d'un « regard intégrateur "; comparant les contextes romand et québécois, les auteurs centrent leur analyse et leurs préconisations à la fois sur les outils, la démarche d’accompagnement et la nécessaire formation des accompagnateurs. Dans le contexte des Hautes Écoles de Suisse romande, en s'appuyant sur la didactique professionnelle et la théorie du "contrat didactique », Barioni et Puozzo font apparaitre l'écart entre l'objet d’apprentissage qu’est censé porter le stage et les attentes et représentations que peuvent en avoir ces stagiaires ; le faire finit par 
y supplanter l'apprendre. Enfin, Guervil et Duchesne ont enquêté auprès d’enseignants en formation en Haïti et en retirent quelques constats saillants; si les enseignants stagiaires reconnaissent l'importance décisive des stages à leur formation et leur entrée dans le métier, ils n’en relèvent pas moins la nécessité de modifier les conditions de leur fonctionnement et particulièrement l’accompagnement des enseignants en stage. Ces trois contributions comportent l'avantage complémentaire de nous permettre de voyager entre différents modèles et surtout différents contextes nationaux de la formation des enseignants.

\section{Références bibliographiques}

Boutinet, J-P. (2011). Un imaginaire contemporain saturé de responsabilité . Éducation permanente, 187, p. 9-21.

Combes, M-C. (2011). La professionnalisation des études universitaires. Éducation permanente, 188, p. 25-35.

De Briant ,V. \& Glaymann, D. (Dir.). (2013). Le stage. Formation ou exploitation? Rennes, Presses Universitaires de Rennes.

Guigou, J. (1975). La stagification. Éducation permanente, 31, p. 3-25.

Lambert, M., Marion-Vernoux, I. \& Sigot, J.C. (2009). Quand la formation continue : repères sur les pratiques de formation des employeurs et des salariés. Enquêtes Céreq, Marseille.

Merle, V. \& Théry, M. (2012). Un projet politique pour les formations en alternance », dans Éducation permanente, 190, p. 9-29. 